\title{
Acoustic metrology for dimensional conformance
}

\author{
John G. Cherng \\ Mechanical Engineering Department, The University of Michigan-Dearborn, 4901 Evergreen Road, Dearborn, \\ MI 48128-1491, USA
}

\begin{abstract}
This study presents an innovative concept - acoustic metrology - for on-line dimensional conformance inspection. It investigates the linear and radial dimensions as well as the true position of a center. It conclusively finds that as these dimensions change, the resonant frequencies of tested cylindrical and flat plate parts also shift. The high resonant frequencies appear to shift more than low resonant frequencies with linear and radial dimensional deviations of cylindrical parts. However, the dimensional deviations of a flat plate part only influence certain numbers of resonant frequencies. The amount of the frequency changes of all tested parts is proportional to the dimensional deviations. The test results reveal a feasible gaging method that can be used for inspection of a production line for a "Go" or "No Go" decision.
\end{abstract}

Key words: Acoustic frequency spectrum; Acoustics metrology; Dimensional conformance; Dimensional inspection

\section{Introduction}

Using an acoustic signal generated by impacting a metallic part to inspect the part's quality traces back to ancient bell makers. An experienced bell maker knows where and how much to grind by listening to the sound of the bell when it is struck. Similar applications are found in current industrial manufacturing processes, such as identifying hydraulic network damages, detecting gear wears, and abnormal machinery operating conditions [1-5]. New adaptive methods have also developed to filter out background noises during the sound measurement [6,7]. These applications are based upon one common principle: resonant frequencies of an elastic object depend on the mass and the stiffness distribution within the object. Thus, whenever changes occur in these properties, resonant frequencies of the object will also shift. Shifts in resonant frequencies are detected by comparing two spectra; one a standard spectrum, and the other a spectrum from the tested part. A large deviation between two spectra represents a defect.

This principle also applies to the dimensional deviations of a mechanical part. As the dimensions of a mechanical part change during the manufacturing process from tool wear or misalignment, the mass and stiffness distribution of the part are altered as well. Consequently, the acoustic frequency spectrum generated by the part is different.

Through a systematic testing and evaluation process, this paper has revealed and found convincing data to support the above application, i.e., dimensional conformance using the acoustic frequency spectrum. This technology can provide many advantages in comparison to other dimensional measuring methods.

(1) Highly efficient: The time required to complete inspection, from impacting the part to signal processing, is less than a few seconds.

(2) Non-destructive: The magnitude of the impact force is just large enough to generate the 
acoustic signal. No damage occurs during inspection.

(3) On-line inspection: Since the time and process are short and no continuous contract is required, the inspection process can be integrated into the manufacturing process as shown in Fig. 1. This offers an opportunity to achieve $100 \%$ quality assurance inspection.

(4) Feedback process control: Computer software can be developed to provide a warning signal or to stop the manufacturing process through a feedback control system when a defective part is detected. This feedback system will ensure the tool is changed in time, before more defective parts are manufactured.

(5) Automatic process: The entire process can be automated without any human involvement after the standard frequency spectrum is established.

Since dimensional accuracy is an essential requirement to the quality of manufactured products, this paper provides a unique approach that results in only quality products being shipped. Another benefit is that this proposed technology can be implemented at a very low cost to almost all types of industries.

\section{Test set-up and test samples}

The set-up of the testing facility is similar to the schematic shown in Fig. 1, except the feedback control system and conveyor are not used. The test facility consists of a B\&K 2034 dual-channel

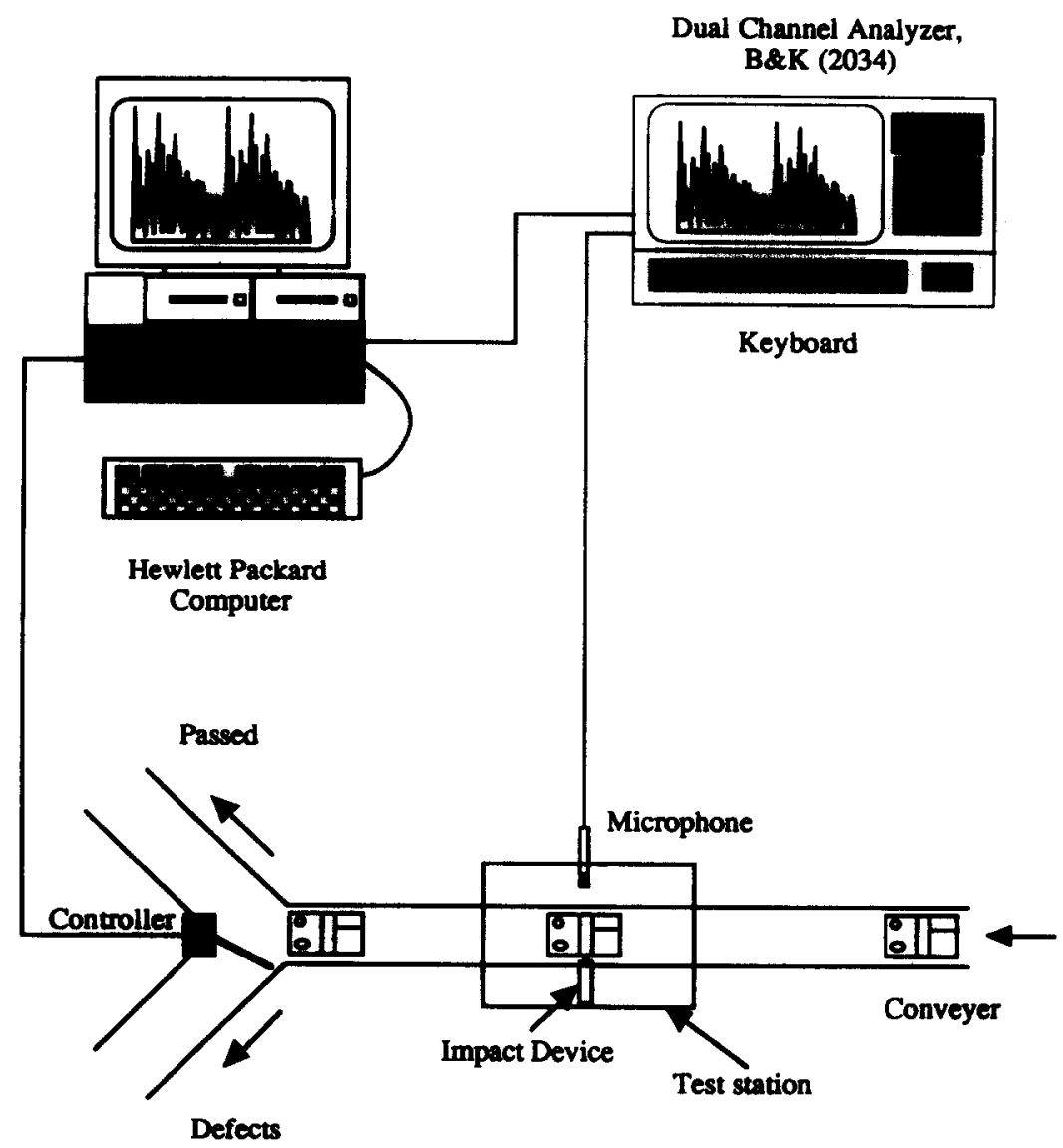

Fig. 1. Schematics of an on-line acoustic inspection station. 
FFT analyzer, a B\&K 4133 high-frequency microphone, an impact device, and an HP 9000/200 computer.

Testing samples are either supported by foam or suspended by rubber bands to achieve a free vibration condition. Test samples that are manufactured in-house cover the following dimensional categories:

\section{(i) Linear dimensional deviation samples}

Three solid aluminum cylinders, $0.5^{\prime \prime}(12.7 \mathrm{~mm})$ in diameter and $4.00^{\prime \prime}(101.60 \mathrm{~mm})$ long, are made. The length of each cylinder is then reduced by $0.010^{\prime \prime}(0.254 \mathrm{~mm})$ each time. The configuration of these solid cylinders is shown in Fig. 2(a).

\section{(ii) Radial dimensional deviation samples}

Two different types of cylinders, one solid (sample A) and one hollow (sample B), are used. The solid cylinders are identical to the above, but the hollow cylinders have the same dimensions with a $0.25^{\prime \prime}(6.35 \mathrm{~mm})$ diameter hole drilled through as shown in Fig. 2(b). For each type of

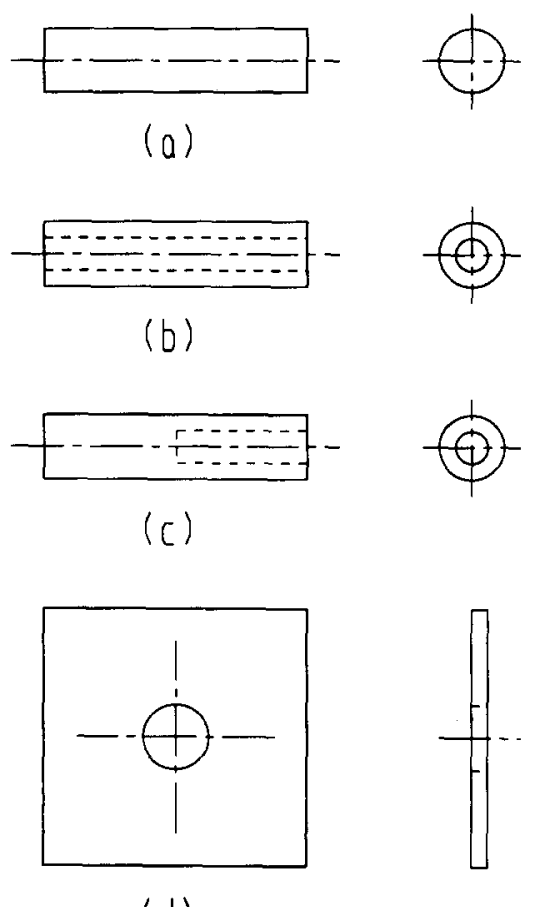

(d)

Fig. 2. Configurations of the test samples. cylinder, three identical parts are made. Two of them have different dimensions, and the third part is kept as the standard part. The outside diameters of the solid and hollow cylinders decrease by $0.010^{\prime \prime}(0.254 \mathrm{~mm})$ each time.

To further investigate the significance of radial dimensional deviations, nine $4.0^{\prime \prime}$ by $4.0^{\prime \prime}$ ( $101.6 \mathrm{~mm}$ by $101.6 \mathrm{~mm}$ ) aluminum plates with a thickness of $0.25^{\prime \prime}(6.35 \mathrm{~mm})$ are made, three of them having a $1.0^{\prime \prime}(25.4 \mathrm{~mm})$ diameter hole drilled at the center (Standard Plate); another three plates with 0.99" (25.146 mm) diameter holes (Plate Type 1); and the last three plates with $0.98^{\prime \prime}$ (24.892 $\mathrm{mm}$ ) diameter holes (Plate Type 2). The configuration of the flat plate is shown in Fig. 2(d). All nine plates are made identically in every aspect except for the hole size, and manually measured before and after the holes are drilled.

\section{(iii) True position of the center of a hole samples}

The deviation of the true position of a center is examined in both axial and radial directions. Changing the depth of a hole in a cylinder produces an axial deviation. The radial deviation is made by moving the center of a drilled hole on a flat plate.

The depth of a drilled hole is difficult to verify by any manual measuring methods. This investigation is conducted by drilling a $0.25^{\prime \prime}(6.35 \mathrm{~mm})$ diameter hole with a $2.0^{\prime \prime}(50.8 \mathrm{~mm})$ depth in a $4.0^{\prime \prime}(101.6 \mathrm{~mm})$ long and $0.5^{\prime \prime}(12.7 \mathrm{~mm})$ diameter solid cylinder as shown in Fig. 2(c). Then the depth of the hole is increased by $0.125^{\prime \prime}(3.175 \mathrm{~mm})$ each time.

The acoustic signals generated by these test samples are much higher than the background noise. The overall sound pressure level of the signal is in the range of 85 to $90 \mathrm{~dB}$. The sound pressure level of the background noise is in the range of 55 to $60 \mathrm{~dB}$. Therefore, the influence of the background noise is not considered in all tests.

\section{Test results and discussions}

\subsection{Linear dimensional deviations}

Fig. 3 shows the spectrum plots of a cylinder whose length has been reduced from $4.00^{\prime \prime}$ 
Solid Cylinder Linear Length $=4.00$ in $(101.60 \mathrm{~mm})$

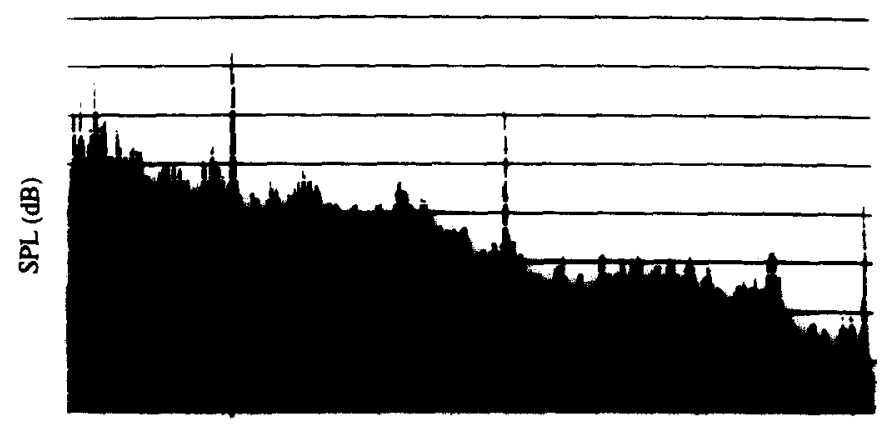

Frequency $(\mathrm{Hz})$

Solid Cylinder Linear Length $=3.99$ in $(101.346 \mathrm{~mm})$

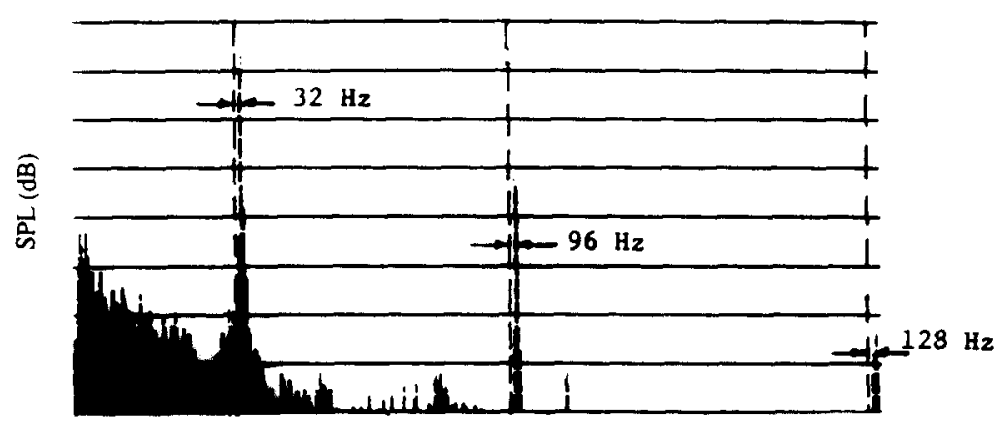

Frequency $(\mathrm{Hz})$

Solid Cylinder Linear Length $=3.98$ in $(101.092 \mathrm{~mm})$

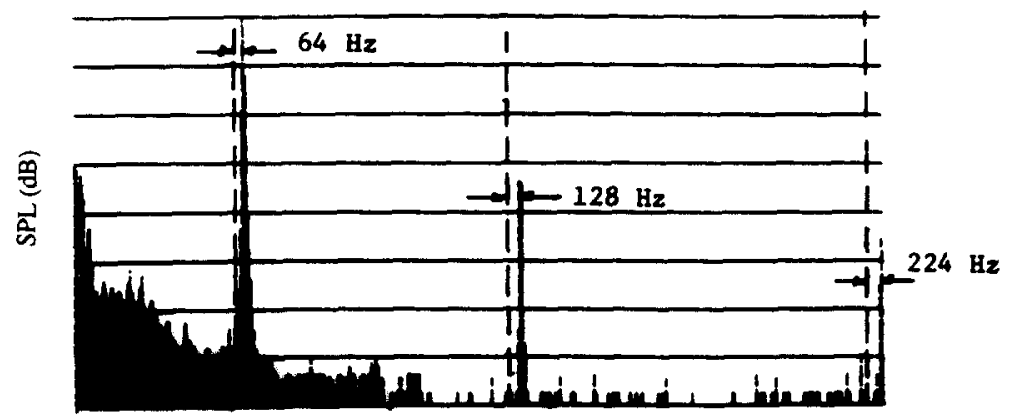

Frequency $(\mathrm{Hz})$

Fig. 3. Spectrum plots of cylindrical parts with linear dimensional deviations. 
$(101.60 \mathrm{~mm})$ down to $3.98^{\prime \prime}(101.092 \mathrm{~mm})$. Three resonant frequencies continuously shift to the right from the original resonant frequencies. The changes in frequency range from $32 \mathrm{~Hz}$ to $64 \mathrm{~Hz}$ for the first peak, $96 \mathrm{~Hz}$ to $128 \mathrm{~Hz}$ for the second peak, and $128 \mathrm{~Hz}$ to $228 \mathrm{~Hz}$ for the third peak. The amount of frequency change is proportional to the linear dimensional deviations. Therefore, the resonant frequencies of the cylinders increase as the masses of the cylinders decrease. This phenomenon is consistent with the fundamental relationship of the natural frequency of an elastic object (the solid cylinder can be considered as a system consisting of a series of discrete masses and springs):

$f_{\mathrm{n}}=\sqrt{k / m}$,

where: $f_{\mathbf{n}}$ is the natural frequency, $k$ is the stiffness, and $m$ is the mass of the object.

Apparently, when the linear dimensions of the tested solid cylinders decrease, the increase in the stiffness of the cylinders and the decrease in mass cause the resonant frequencies to increase.

Fig. 4 plots frequency changes for each peak versus the linear dimensional deviations of all these cylinders. The lengths of the cylinders are reduced from $4.0^{\prime \prime}(101.60 \mathrm{~mm})$ to $3.97^{\prime \prime}(100.838 \mathrm{~mm})$, i.e. deviations from $0.0^{\prime \prime}(0.0 \mathrm{~mm})$ to $0.03^{\prime \prime}(0.762 \mathrm{~mm})$. The resonant frequencies increase from 32 to $96 \mathrm{~Hz}$ for the first peak, 96 to $212 \mathrm{~Hz}$ for the second peak, and 128 to $332 \mathrm{~Hz}$ for the third peak. The test data are indicated by four alphabetical and numerical letters. The first two letters are for the cylinder number, e.g. " $\mathrm{C} 1$ " is cylinder 1 and " $\mathrm{C} 2$ " is cylinder 2. The last two letters are for the peak number, e.g. "P1" is the first peak and "P2" is the second peak. Therefore, "C1P1" is the first peak of cylinder 1 . In addition, a positive change is used for a resonant frequency increase and a negative change is used for a frequency decrease. All subsequent figures of this paper will use this numbering system.

It can be seen that all three tested cylinders have almost identical frequency changes for a same amount of linear dimensional deviation. The consistency of the test results from all tested cylinders provides a strong support to the process.

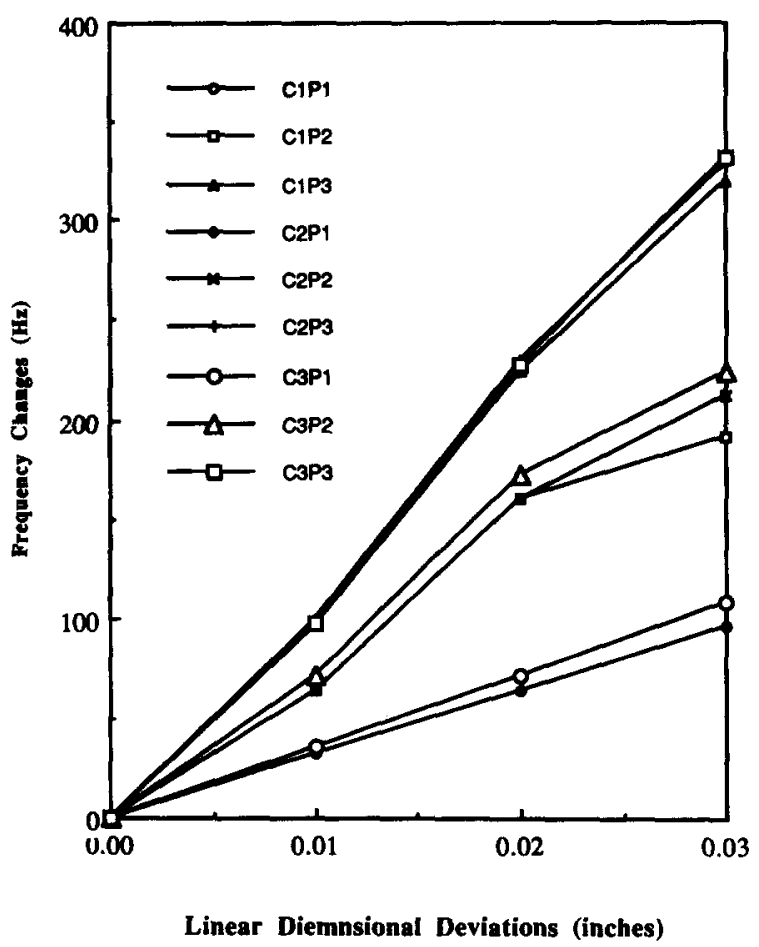

Fig. 4. Changes of resonant frequencies of cylindrical parts versus linear dimensional deviations.

\subsection{Radial dimensional deviations}

Spectrum plots of a solid cylinder with diameter changes from $0.50^{\prime \prime}(12.7 \mathrm{~mm})$ to $0.49^{\prime \prime}$ $(12.446 \mathrm{~mm})$ are shown in Fig. 5 . The frequency decreases by $112 \mathrm{~Hz}$ for the first peak and $529 \mathrm{~Hz}$ for the second peak. However, this time the peak shifts to the left, i.e., decreases in frequency as the diameter changes. Apparently, a change in the outer diameter causes the stiffness to decrease more than a decrease in mass. Therefore, the resonant frequencies of tested parts decrease as the diameters decrease. Figs. 6 and 7 show the frequency changes with respect to diameter deviations of the solid (indicated by "S") and hollow cylinders (indicated by " $H$ ") respectively. The outer diameters reduce from $0.5^{\prime \prime}(12.7 \mathrm{~mm})$ to $0.47^{\prime \prime}$ $(11.938 \mathrm{~mm})$. The frequency changes from $-112 \mathrm{~Hz}$ to $-288 \mathrm{~Hz}$ for the first peak, and from $-529 \mathrm{~Hz}$ to $-961 \mathrm{~Hz}$ for the second peak. The third peak is not used because it is not substantially above the adjacent readings after the diameter 


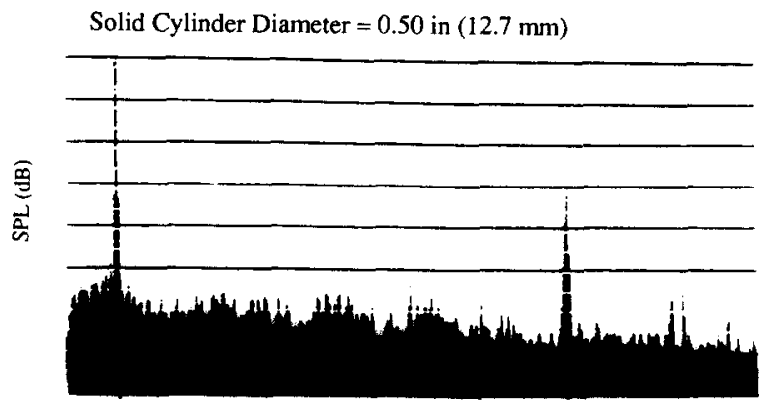

Frequency $(\mathrm{Hz})$

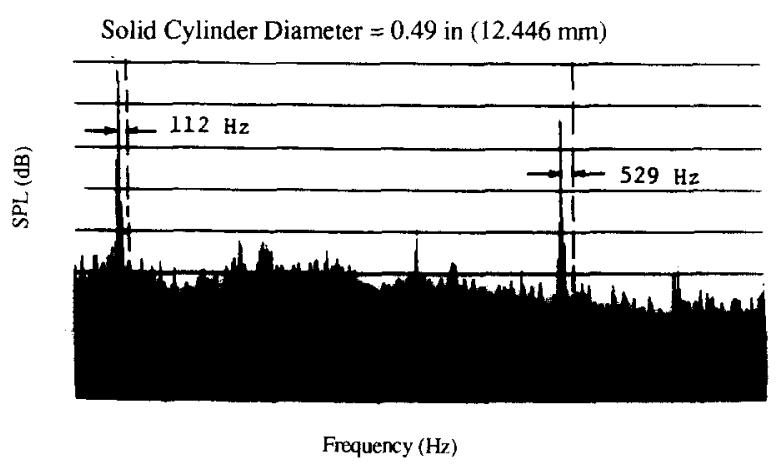

Fig. 5. Spectrum plots of cylindrical parts with radial dimensional deviations.

changes. Therefore, the radial dimensional deviations cause a greater change in resonant frequencies than the linear dimensional deviations. This also implies that the stiffness is more sensitive to the radial dimensional changes than the linear dimensional deviations for a cylindrical part.

The frequency spectrum of the flat plate is very different from the cylinder's spectrum, as shown in Fig. 8. Instead of having only a few peaks as with the cylinders, the plates with a hole show a total of eleven peaks. However, as the diameter of the hole changes, not all the resonant frequencies shift. Fig. 9 shows the changes in all resonant frequencies of Plate Type 1 and Plate Type 2 with smaller diameter holes than the Standard Plate. Averaged values of the frequency changes are used in the plots because different plates were used for different deviations. The resonant frequencies shift most at the sixth, ninth, and tenth resonant frequencies for Plate Type 1. And additionally, the fourth, fifth, seventh and eighth resonant frequen-

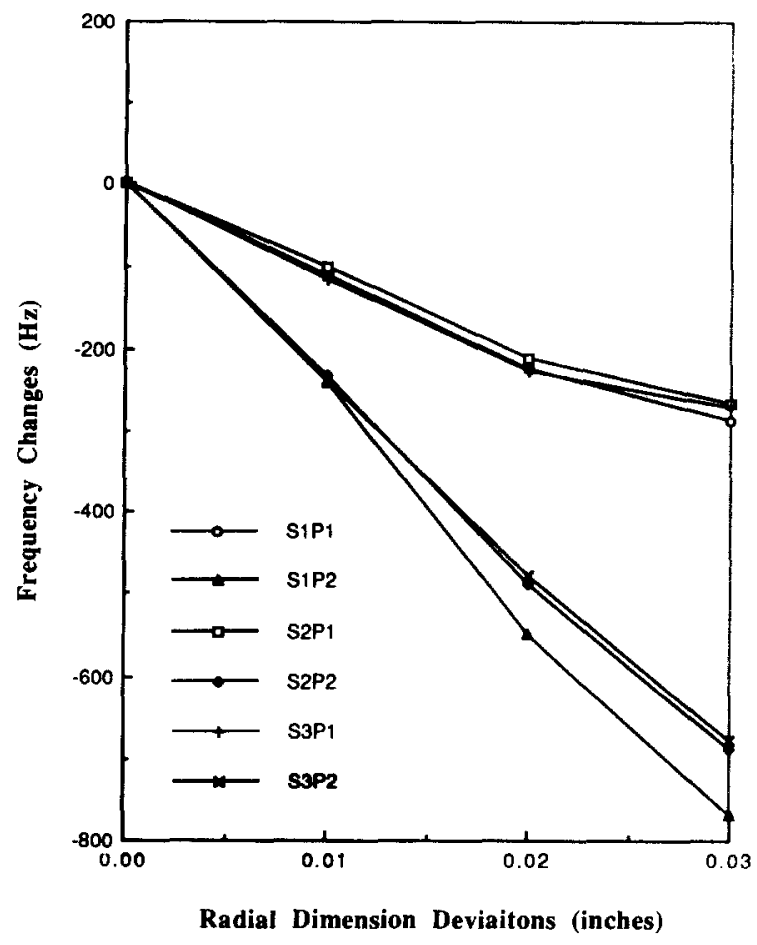

Fig. 6. Changes of resonance frequencies of solid cylindrical parts versus radial dimensional deviations.

cies have also shown substantial frequency shifting for Plate Type 2 as the hole size become smaller.

In general, all resonant frequencies of the plates decrease as the diameter of the hole decreases, i.e., as the mass of the plates increases. Furthermore, the deviations of the radial dimension of the hole in a flat plate have stronger influence on some certain resonant frequencies than others, instead of on every resonant frequency as in a cylindrical part. Depending on the real tolerance control range and application, one can specify certain resonant frequencies for dimensional conformance inspection. For example, if the tolerance is below $0.01^{\prime \prime}(0.254 \mathrm{~mm})$, the sixth, ninth, and tenth resonant frequencies should be used. If the tolerance is increased to $0.02^{\prime \prime}(0.508 \mathrm{~mm})$, then the fourth to tenth resonant frequencies could be chosen as references. A computer program has been developed to demonstrate the procedures of using certain resonant frequencies. This is discussed in a later section. 


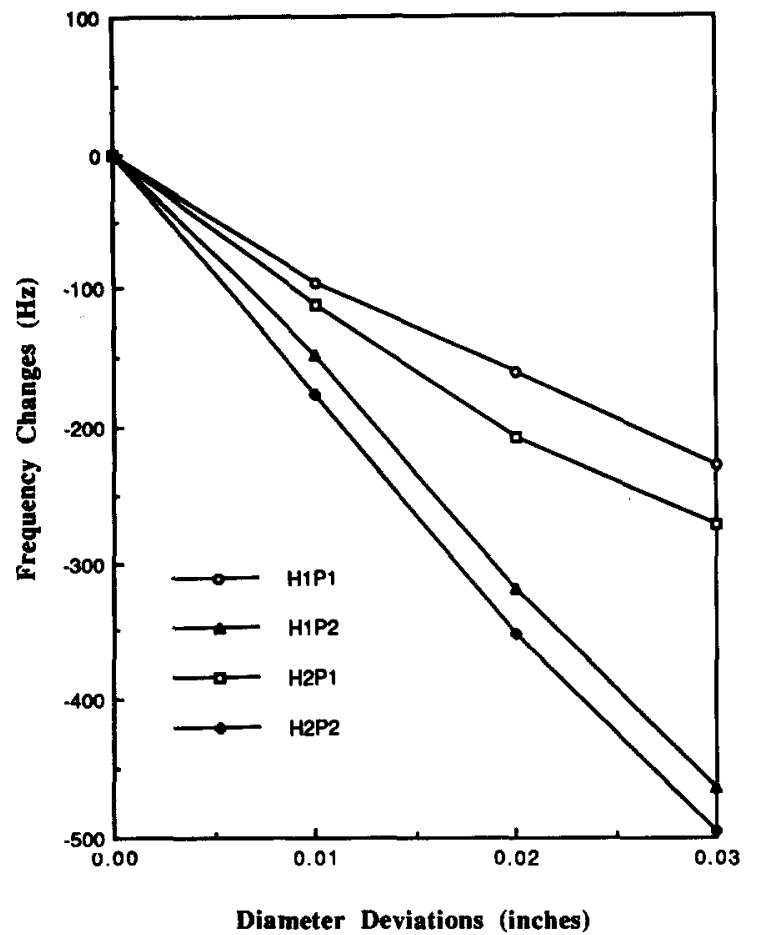

Fig. 7. Changes of resonant frequencies of hollow cylindrical parts versus radial dimensional deviations.

\subsection{True position of a center}

The axial deviation of the true position of a center was investigated by changing the depth of a hole in a cylindrical part. Fig. 10 shows frequency changes as the depth of the hole increases. Both

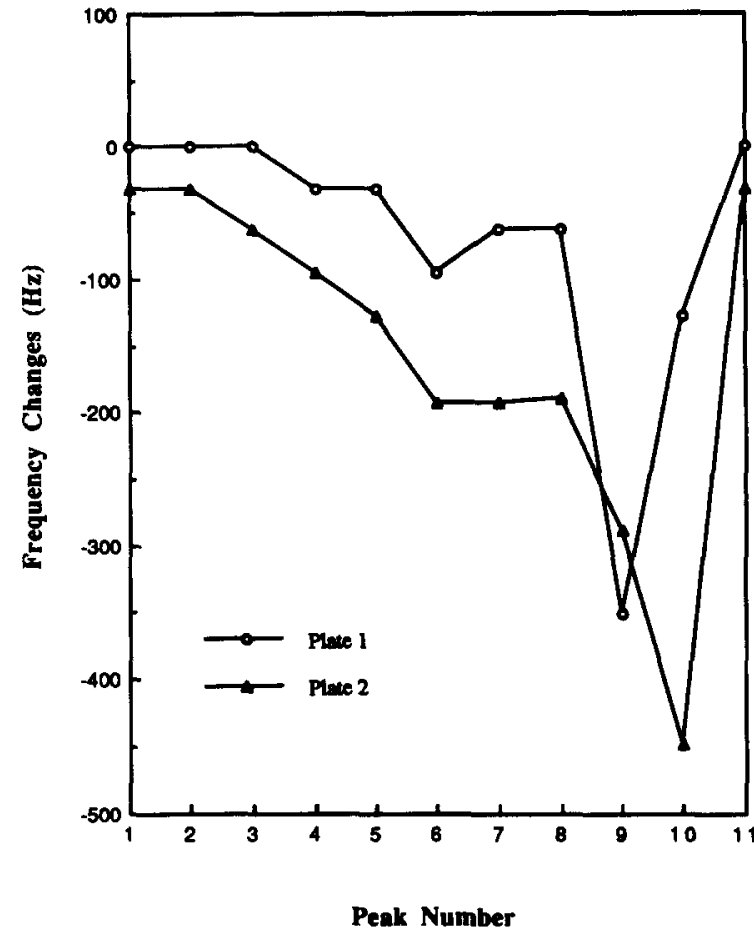

Fig. 9. Changes of resonant frequencies of flat plates with a hole.

resonant frequencies of the cylinders decrease as the depth of the hole increases, i.e., the changes of resonant frequencies are all negative. Once again, the stiffness of the cylinder is more affected by the axial deviation of the center of the hole than the mass of the cylinder.

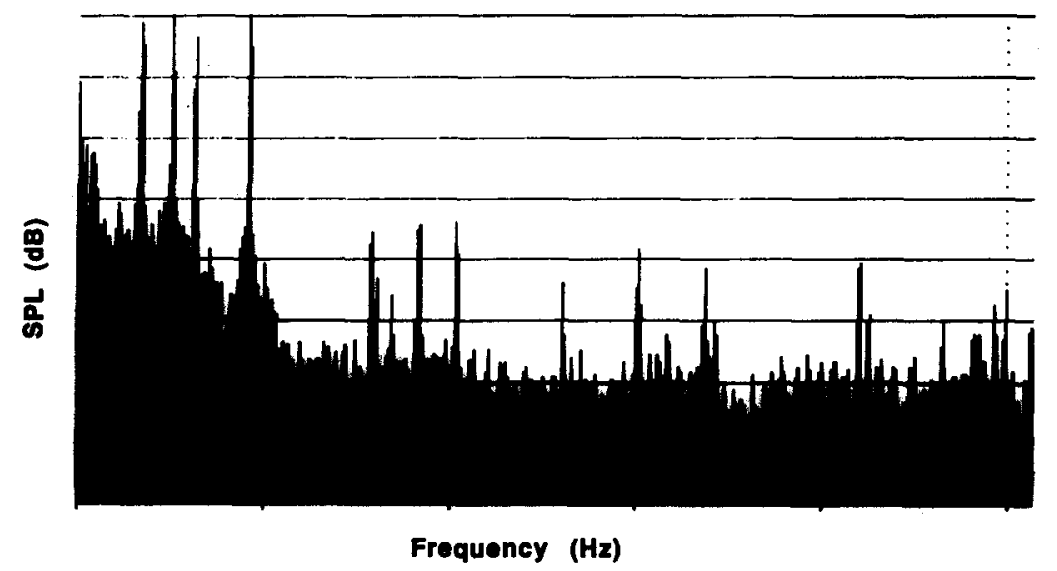

Fig. 8. Acoustic spectrum of a flat plate with a hole. 


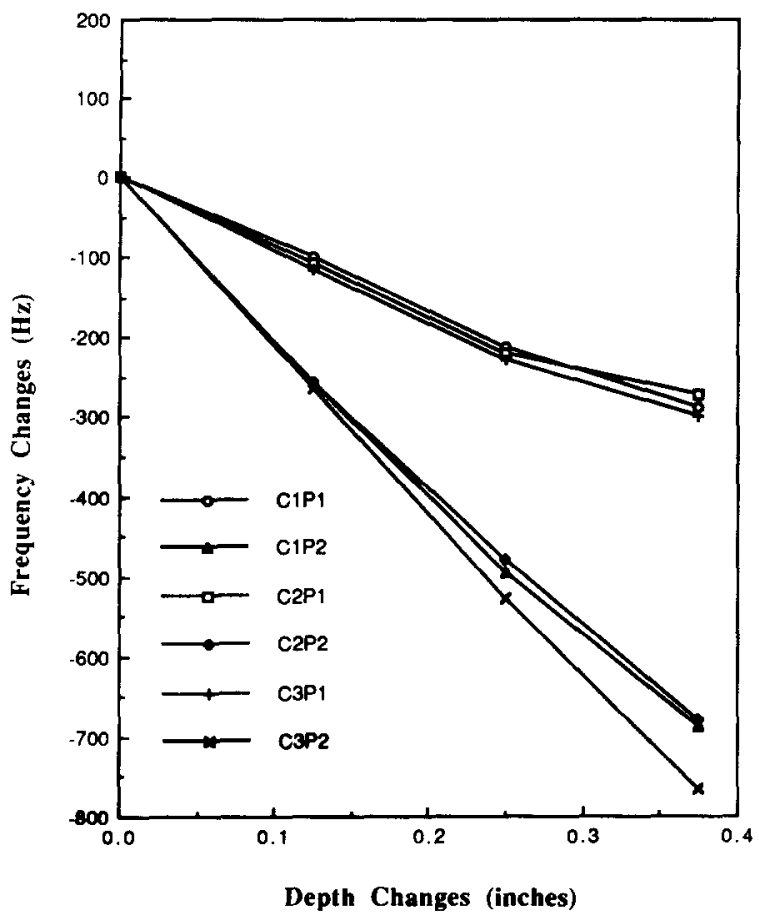

Fig. 10. Changes of resonant frequencies of cylindrical parts versus the deviation of the depth of the hole.

The radial deviation of the true position of a center was investigated by altering the center of a hole on a flat plate. Two types of plate are manufactured. Plate 1 is the standard plate. Plate 2 is the plate with the location of its center altered by $0.010^{\prime \prime}(0.254 \mathrm{~mm})$ in one direction. Fig. 11 plots the shifts of all resonant frequencies of three plates of Type 2. The most significant shifts are at the sixth, ninth, tenth and eleventh peaks. Apparently, the true position of the center has a strong effect on certain high resonant frequencies. These highfrequency peaks can be identified in the preliminary tests and be used as the reference resonant frequencies for on-line inspection purposes.

Most of the frequency changes are negative except the fifth peak. This may indicate that these changes are mainly due to the stiffness reduction instead of the mass change. Although the size of the hole has not changed, the deviation of the center may cause the mass distribution of the plate to be altered. If we consider the plate as a series of discrete masses and springs, then some portions of the plate may increase in mass and other

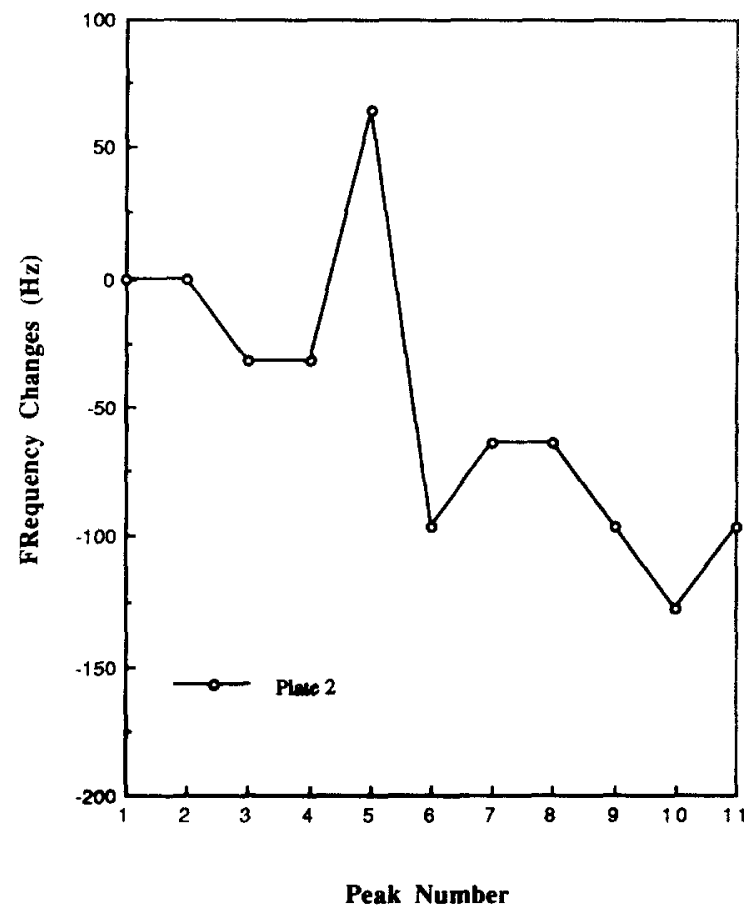

Fig. 11. Changes of resonant frequencies of a flat plate due to the deviation of the true position of the center of a hole.

portions of the plate may decrease in mass due to the deviation of the center. A similar situation may apply to the stiffness distribution, i.e. some portion may increase in stiffness and another portion may decrease in stiffness. However, since most of the frequency changes are negative, either the portions with decreased mass are not dominant in the frequency change, or the stiffness reduction in certain portions of the plate has dominated the dynamic behavior of the plate.

\section{Software development}

A computer program has been developed to conduct the evaluation of the tested sample's acoustic frequency spectrum. The program is written in HPBASIC and has an interactive user interface.

The operation of the software consists of several steps. At first, a standard part and a faulty part are tested. The acoustic frequency spectra are 
analyzed and the resonant frequencies that are critical for dimensional conformance are selected.

When the produced parts are ready for inspection, one can simply type "QC" (stands for Quality Control) at the DOS environment to activate the program. As the software is executed, the user is prompted to enter the number of resonant frequencies and their operating and control ranges. The operating range is the range that the software will search for each resonant frequency. The control range is the lower and upper limits of each resonant frequency. Then, the program asks for the criteria for "Passed" and "Failed" of each test. One can select either 100 percent, which requires that all the resonant frequencies of the tested part must be within the control range of each resonant frequency, or other values less than 100 percent.

When a part is struck, the software first reads in all sound pressure levels of the entire frequency spectrum and then searches for the peak values in each operating range to determine the resonant frequencies. The resonant frequencies will be compared with the lower and upper limits of each control range. The program counts the total number of resonant frequencies that either exceed the upper limit or lie below the lower limit. Finally, the percentage of total resonant frequencies that are within the control range is calculated. If the percentage value is greater or equal than the criteria for the given test parts, a "Passed" message is displayed. Otherwise, a "Failed" message is shown.

All samples were repeatedly tested with the software. The software was able to distinguish all faulty parts without any errors.

\section{Conclusions}

The test results are highly supportive of the concepts presented in this study. The following promising conclusions result from the above evaluations:

(1) Test results indicate that acoustic resonant frequencies of an elastic part are closely related to its geometric dimensions and can be used for dimensional conformance inspections.

(2) The resonant frequency changes appear pro- portional to the dimensional deviations of the linear, radial and the true position of a center. In addition, as geometric dimensions change, either linearly or radially, higher resonant frequencies appear to shift more than lower resonant frequencies.

(3) When an object, such as a flat plate with a hole, that has many resonant frequencies is struck, changes of the resonant frequencies may occur at only a few frequencies. One should identify the critical resonant frequencies for a specific geometric dimensional control.

(4) It appears that the deviations in the geometric dimensions cause the revisions in the mass and stiffness distributions of a metallic part. The changes of the resonant frequencies, either increase or decrease, are determined by which distribution is more affected by the geometric deviations.

(5) It is believed that the precision and accuracy of the process is highly dependent on the resolution of the frequency spectrum. Since the highfrequency resonances are significant to the inspection, the maximum range of the frequency spectrum may have to be set at a higher value in order to capture the high-frequency resonances. As the maximum frequency range gets larger, the bandwidth of each frequency band also increases. Because the frequency change is proportional to the dimensional deviation, the amount of frequency change due to a very small dimensional deviation may not be seen in a low-resolution frequency spectrum. For example, if the maximum frequency range is set at $25.6 \mathrm{kHz}$, the bandwidth of each line on the screen will be $32 \mathrm{~Hz}$ for an analyzer with a resolution of 800 lines. Therefore, if the frequency change is less than $32 \mathrm{~Hz}$, it will not be identified on this frequency spectrum. For the same reason, a constant-bandwidth analyzer, such as the FFT analyzer used in this study, is definitely more desirable than a constantpercentage bandwidth analyzer, where the bandwidth increases as the center frequency increases.

(6) The effects of variables other than dimensions, e.g. material differences, cavities, impurities, etc., on the accuracy of the process needs to be determined. However, it is believed that the deviations in other variables from the standard part can be detected simultaneously, since deviations on 
these variables will most likely also change the mass and/or stiffness distribution of the tested part. Such changes consequently shift the resonant frequency, thereby allowing for detection through the presented method. This continues to fulfill the objective of the process for a "Go" or "No Go" inspection.

\section{Acknowledgements}

The research presented in this paper is supported in part by the Research Excellence and Economic Development Fund, State of Michigan under contract number 238568 . The author is very grateful for the encouragement given by the program directors, Dr. William Spurgeon and Dr. Fredric G. Bolling at the University of Michigan-Dearborn.

\section{References}

[1] P.A. Drakatos and S.P. Draketos, The use of noise and vibration on the hydraulic network, in: Structural Vibration and Acoustics, ASME DE-Vol. 34, 1991, pp. 159-160.

[2] P.A. Drakatos, Diagnosis and Prognosis, MIT Press, Cambridge, MA, 1982.

[3] R.A. Collacot, Mechanical Fault Diagnosis and Condition Monitoring, Chapman and Hall, London, 1977.

[4] H. Tang, J.Z. Cha, Y. Wang and C. Zhang, The principle of cepstrum and its application in qualitative fault diagnostics of gears, in: Modal Analysis, Modeling, Diagnostics, and Control-Analytical and Experimental, ASME DE-Vol. 38, 1991, pp. 141-144.

[5] R.D. Randall, Cepstrum analysis and gearbox fault diagnosis, B\&K Application Note, No. 233-8, 1988.

[6] G.P. Bosworth, et al., Noise analysis of automotive components utilizing adaptive filtering techniques. Hughes Aircraft Company, Fullerton, CA.

[7] B. Widrow, et al., Adaptive noise canceling: Principles and applications, Proc. IEEE 63(12) (1975). 